Revista de Psicología Vol. 36 (2), 2018 (ISSN 0254-9247)

\title{
La contribución del MMPI-2 a la predicción del riesgo de violencia
}

\author{
Amada Ampudia Rueda ${ }^{1}$, \\ Guadalupe Sánchez Crespo², Fernando Jiménez Gómez ${ }^{3}$ \\ Universidad Nacional Autónoma de México ${ }^{1}$, \\ Universidad de Salamanca, España $a^{2,3}$
}

El presente estudio analiza la contribución del MMPI-2 en la predicción del riesgo de violencia. Un total de 574 participantes componen los dos grupos ya establecidos: el grupo "Homicida", integrado por 287 reclusos condenados por homicidio que están cumpliendo condena en diversas cárceles del Distrito Federal y del Estado de México, y el grupo "Nodelictivo", formado por 287 participantes que no cometieron delito alguno, siendo un total de 215 varones y 72 mujeres en cada grupo. Se analizaron sus diferencias de medias, su tamaño del efecto ( $\mathrm{d}$ de Cohen) y la precisión diagnóstica aportada por la curva ROC en el conjunto de escalas del MMPI-2. Los resultados muestran las escalas con mayor incidencia potencial del riesgo de violencia.

Palabras clave: homicidio, MMPI-2, violencia, curva ROC.

\section{The contribution of the MMPI-2 to the prediction of the risk of violence}

The present study analyzes the contribution of the MMPI-2 in the prediction of the risk of violence. 574 participants compose the two groups: the "Homicidal" group, comprising 287 prisoners convicted of homicide and serving sentences in several prisons in Mexico City, and the "Non-criminal" group, comprising 287 participants who did not commit any crime. A total of 215 men and 72 women are in each group. Differences in means were analyzed and effect sizes (Cohen's d) were calculated. The ROC curve is used in the set of scales of the MMPI-2 to assess diagnostic accuracy. The results present the scales with greater potential impact of the risk of violence.

Keywords: homicide, MMPI-2, violence, ROC curve.

1 Doctora en Psicología por la Universidad Nacional Autónoma de México. Dirección postal: Av. Universidad, Av. de los Insurgentes Sur, Copilco Universidad, Coyoacán, 04360 Ciudad de México, D.F. Contacto: amada@servidor.unam.mx

2 Doctora en Filosofía y Ciencias de la Educación por la Universidad de Salamanca. Dirección postal: Av. de la Merced, 101.37005.- Salamanca, España. Contacto: lupes@usal.es

3 Doctor en Filosofía y Letras (Sección Psicología) por la Universidad Pontificia de Salamanca. Dirección postal: Av. de la Merced, 101.37005.- Salamanca, Espańa. Contacto: fjimenez@ usal.es 


\section{A Contribuiçáo do MMPI-2 para a prediçáo do risco de violência}

O presente estudo analisa a contribuiçáo do MMPI-2 na predição do risco de violência. Um total de 574 participantes compóem os dois grupos estabelecidos: o grupo "Homicida" é composto por 287 presos condenados por homicidios, que cumprem sentenças em várias prisōes do Distrito Federal e do Estado do México, e o grupo "Não-criminoso" é formado por 287 participantes que não cometeram nenhum crime, sendo um total de 215 homens e 72 mulheres em cada grupo. Foram analisadas as diferenças nas médias, o tamanho do efeito ( $\mathrm{d}$ de Cohen) e a precisão diagnóstica fornecida pela curva ROC no conjunto das escalas de MMPI-2. Os resultados mostram as escalas com maior incidência potencial de risco de violência.

Palabras-chave: homicidio, MMPI-2, violência, curva ROC.

\section{La contribution de MMPI-2 à la prédiction du risque de violence}

La présente étude analyse la contribution de MMPI-2 dans la prédiction du risque de violence. Au total, 574 participants constituent les deux groupes déjà constitués: le groupe "Homicide", composé de 287 détenus condamnés pour homicide qui purgent des peines dans diverses prisons du District fédéral et de l'État de Mexique, et du groupe "Non-criminel", formé par 287 participants qui n'ont commis aucun crime, soit un total de 215 hommes et 72 femmes dans chaque groupe. Leurs différences de moyennes, leur taille d'effet (Cohen d) et la précision diagnostique fournie par la courbe ROC dans l'ensemble des échelles MMPI-2 ont été analysées. Les résultats montrent les échelles avec l'incidence potentielle la plus élevée du risque de violence.

Mots-clés: homicide, MMPI-2, violence, courbe ROC. 
La violencia es un fenómeno muy complejo de predecir y delimitar, tanto por su origen multicausal como por sus numerosas variables y diversidad de expresiones implicadas. Existen diversos criterios para predecir adecuadamente una conducta como "potencialmente violenta" siguiendo criterios de personalidad, psicopatológicos, delictuales (Gutiérrez \& Portillo, 2014), motivacionales, psicosociales e, incluso, de reincidencia (Jiménez et al., 2014; Ráez \& Martínez, 1985). Andrés Pueyo y Redondo (2007) distinguen cinco propiedades que caracterizan a la violencia: es compleja, multicausal, heterogénea, intencionada e infrecuente. En los últimos años, el foco de las diversas polémicas suscitadas ha estado centrado no tanto en poder predecir un acto violento, sino cómo valorar los grados de riesgo que presenta. Para la OMS la violencia consiste en "el uso deliberado de la fuerza física o el poder, ya sea en el grado de amenaza, o efectivo, contra uno mismo, otra persona, grupo o comunidad, que cause o tenga muchas probabilidades de causar lesiones, muerte, dańos psicológicos, trastornos del desarrollo o privaciones" (Krug et al., 2002, p. 5).

La peligrosidad es un término que se ha venido utilizando hasta los ańos 90 y es bien entendido por la práctica jurídica y por los profesionales de salud mental; sin embargo, se tiende a sustituir por el de valoración del riesgo de violencia. Aunque los dos términos persiguen la misma finalidad, se diferencian por su justificación, eficacia y las ventajas para los propios profesionales (Pueyo \& Redondo, 2007; Jiménez et al., 2014). No obstante, esta predicción del riesgo de la conducta violenta no parece fácil. Independientemente de la polémica planteada por los defensores (Derzon, 2001; Loza, 2003; Megargee, 1976; Monahan, 1981) y detractores (Grisso \& Appelbaum, 1992) de la predicción, o de cuáles son los factores que pueden estar inmersos en la conducta violenta, lo que sí se confirma es la existencia de un consenso general de que los evaluadores deberían contemplar la violencia desde diversas 
fuentes, tanto biográficas y clínicas, como sociodemográficas (Heilbrum, 2009; Soria et al, 2009) y también de reincidencia (Webster, Douglas, Eaves \& Hart, 1997b). La estrategia metodológica utilizada para evaluarla (Actuarial o Clínica) es otra polémica generada en la investigación de la predicción del riesgo de violencia.

Teniendo como base los tests psicológicos, la metodología actuarial se caracteriza por ser un procedimiento psicométrico en el que se introducen las variables relevantes, ponderadas o no, con el objetivo de determinar la capacidad predictiva del instrumento. Este procedimiento actuarial tiene, como esencia, las características de ser sistemáticos, asépticos e imparciales, minimizando el juicio clínico y disponiendo de una gran validez y fiabilidad (Cunningham, Sorensen \& Reidy, 2005; Webster, Hucker \& Bloom, 2002). Algunos estudios (Gardner, Lidz, Mulvey \& Shaw, 1996; Torrubia, 2004) sugieren que estos procedimientos actuariales poseen una eficacia predictiva superior a los procedimientos clínicos. Por otra parte, la metodología clínica se basa, fundamentalmente, en criterios epidemiológicos, históricos o de personalidad, tomando como instrumentos esenciales la Observación y Entrevista. Si este tipo de evaluación conservase una información estructurada, fijada previamente y pormenorizadamente detallada en sus componentes esenciales, se denominaría clínica estructurada (Otto, 2000).

Una importante diferencia entre la metodología en las investigaciones realizadas sobre la peligrosidad en los años 70 y la valoración del riesgo de violencia en los albores del siglo XXI, se debe no solo a la influencia de Meehl (1954) al distinguir entre "métodos de predicción clínica" y "actuariales o estadísticos", sino también a la Teoría de la Decisión. El análisis a través de la curva ROC (Receiver Operating Characteristic) se llevó a cabo por ser un método discriminante para la precisión diagnóstica de los instrumentos de evaluación tras ser una metodología desarrollada en el seno de la Teoría de la Decisión en los años 50 (Swets \& Pickett, 1982), inicialmente diseñada para la detección de señales por radar y, posteriormente, aplicada en el área de la biomedicina (Zweig \& Campbell, 1993). 
A pesar de las dificultades inherentes en la predicción de los actos violentos por medio de instrumentos de personalidad, algunos investigadores han intentado relacionar las puntuaciones en las escalas del Minnesota Multiphasic Personality Inventory (MMPI) de Hataway y McKinley (1967) con la agresión y el comportamiento antisocial. Es a partir de la década de 1940, cuando el MMPI fue considerado como un instrumento eficaz para el examen de los problemas de salud mental o de personalidad en personas que se someten a investigaciones criminales o ser evaluado para distintas funciones carcelarias (Butcher, Hess, Greene \& Nelson, 2015; Butcher, 2013; Megargee, 1977, 2006a, 2006b). Con la técnica del MMPI se ha investigado una amplia variedad de temas dentro de la población reclusa: características de su personalidad (Fry, 1949), evaluación de la salud mental (Guy, Platt, Zwerling \& Bullock, 1985), reincidencia (Pavelka, 1986), peligrosidad (Nieberding, Moore y Dematatis, 2002), violencia de los delincuentes (Jones, Beidleman \& Fowler, 1981), personalidad de los asesinos (Ampudia, Sánchez \& Jiménez, 2017; Pennuto, 2010) o delincuentes sexuales (Grover, 2011; Lanyon, 1993; Rader, 1977), para ayudar a las instituciones penitenciarias a entender, mejorar y adecuar su trabajo. Pero la agresión no solo se encuentra en los delincuentes, también puede mostrarse en pacientes psiquiátricos y ser necesaria una estrecha supervisión (Eronen, Hakola \& Tiihonen, 1996).

¿Cuáles son las escalas que mejor pueden definir el riesgo de violencia? Jones, Beidleman y Fowler (1981) realizaron un estudio con 141 sujetos adultos, violentos y no violentos, internos en cárceles de máxima seguridad, a los que se les administró el MMPI. Estos autores prefirieron utilizar el análisis discriminante al de los "tipos de código", obteniendo así las escalas que más contribuyeron a predecir su conducta violenta: la F (Infrecuencia), Pa (Paranoia), Pt (Psicastenia) y Sc (Esquizofrenia), clasificando correctamente al $72.9 \%$ de los violentos y al $80.6 \%$ de los internos no violentos. Por su parte, Boscan et al. (2002), en el estudio realizado con delincuentes y no-delincuentes mexicanos, al aplicarle el MMPI-2, encontraron, además de la F (Infrecuencia) y la Sc (Esquizofrenia), la escala de alcoholismo de MacAndrew (Mac-R). 
El estudio elaborado por Megargee, Mercer y Carbonell (1999) con delincuentes, varones y mujeres incide en las escalas de Infrecuencia (F), Desviación Psicopática (Pd), Paranoia (Pa) e Hipomanía (Ma), a la par con la escala de Alcoholismo (Mac-R), la de Prácticas antisociales (ASP) y la de Admisión de adicción (APS) en las mujeres. También las escalas Pa (Paranoia), Pt (Psicastenia), Sc (Esquizofrenia), $\mathrm{Ma}$ (Hipomanía) y F (Infrecuencia) fueron subrayadas por los investigadores (Panton, 1959, 1962b; Oliver \& Mosher, 1968) por presentar mayor incidencia en individuos violentos.

Butcher et al. (2015) resumen los hallazgos de los investigadores con el MMPI-2 que "tienen un mayor riesgo de comportamiento violento: la esquizofrenia, manía, trastorno de la personalidad, el abuso de sustancias, y otras condiciones más raras de la lesión cerebral orgánica y la enfermedad de Huntington" (pp. 210-211). En el conjunto de investigaciones, se observa que la mayor parte de los estudios realizados se han referido fundamentalmente al análisis de las escalas Clínicas básicas, con excepción de alguna referencia a las escalas de Validez y al consumo de Alcohol y/o sustancias como posibles catalizadores del riesgo de violencia. En este estudio se han analizado también las escalas de Contenido y Suplementarias con el objetivo de completar las posibilidades diagnósticas de la técnica de Minnesota. Por tanto, el objetivo de este estudio es analizar la contribución a la predicción del riesgo de violencia con las diversas escalas del MMPI-2, a través del análisis de sus diferencias y la precisión diagnóstica (curva ROC) que presentan sus escalas entre los dos grupos: "Homicidas" y personas que no cometieron delito alguno ("No-delictivo").

\section{Método}

El diseño empleado en este estudio es referido por las técnicas estadísticas de análisis descriptivo, comparación de medias y tamaño del efecto de Cohen (1988) entre reclusos homicidas encarcelados en distintas instituciones del Distrito Federal y estado de México, y entre 
jóvenes no-delictivos, a través de las diversas variables de personalidad evaluadas por el MMPI-2. Se añadió el análisis de la presión diagnóstica de las diferentes escalas del MMPI-2 a través de los índices de la curva ROC.

\section{Participantes}

El total de 574 participantes en este estudio fueron equiparados en número y en género. De ellos, 287 (215 varones y 72 mujeres) son personas sentenciadas por homicidio e internas en diversas instituciones penitenciarias mexicanas, formando el grupo denominado como "Homicidas"; otras 287 (215 varones y 72 mujeres) son jóvenes mexicanos que no han cometido delito alguno y que forman el grupo de "No-delictivo". Los 215 varones del grupo de Homicidas tienen una media de edad de $32.11(D E=9.30)$, con un rango de edad entre $19 \mathrm{y}$ 67 ańos; y las 72 mujeres Homicidas presentan una media de edad de $33.50(D E=7.94)$ con un rango de edad entre 19 y 50 ańos. Los 215 varones del grupo No-delictivo presentan una media de edad de 29.07 años $(D E=8.61)$ con un rango de edades entre 19 y 59 años. Las 72 mujeres de este grupo muestran una media de edad de $29.22(D E=$ 5.94) y un rango de edad entre 23 y 45 ańos. El grupo de Homicidas se encontraba internado en diversos reclusorios del Distrito Federal y estado de México.

Con respecto al nivel de estudios máximo alcanzado por los participantes, $19.51 \%$ de los Homicidas consiguieron terminar la primaria (todos los No-delictivos superaron la Primaria), un 47.74\% finalizó la secundaria ( $5.923 \%$ el grupo de No-delictivo), $25.78 \%$ de los Homicidas finalizó el Bachillerato (19.16 \% el grupo de No-delictivo), $6.62 \%$ del grupo de Homicidas tenían estudios que les habilitaba para ejercer una profesión (58.89\% tenían el grupo No-delictivo) y solamente el $0.35 \%$ de los Homicidas tenían estudios de posgrado finalizado (16.03\%, el grupo No-delictivo).

Con respecto al estado civil, el 32,75\% de los Homicidas están solteros (45.3\%, el grupo No-delictivo), 26.48\% de los Homicidas están casados (49.48\%, el grupo de No-delictivo), 23.69\% están en 
"unión libre" (2.787\%, el grupo de No-delictivo) y el resto de estados (divorciados, separados o viudos) eran de escasa incidencia (homicidas separados: $8.362 \%$ [0.349\% grupo No-delictivo]; homicidas divorciados: $3.484 \%$ [2.091\% grupo No-delictivo] y homicidas viudos: $5.226 \%$ [ $0 \%$ en el grupo No-delictivo]).

\section{Medición}

Para poder llevar a cabo el perfil psicológico nos hemos servido del test de Personalidad de Minnesota (Minnesota Multiphasic Personality Inventory-2; MMPI-2) de Hathaway y McKinley, en su adaptación mexicana (Lucio, Reyes-Lagunes \& Scott, 1994) en su conjunto de Escalas de Validez, Clínicas básicas, de Contenido y Suplementarias. Los coeficientes de confiabilidad alfa resultaron ser bastante altos en la población mexicana tanto en lo que se refiere a las escalas Clínicas básicas (.66), como las de Contenido (.67) y las Suplementarias (.73), (Lucio, Pérez \& Ampudia, 1997). Con diferencias obtenidas con las escalas Clínicas básicas (.03), las de Contenido (.01) y las Suplementarias (.04) en comparación con la baremación estadounidense presentan diferencias muy pequeñas y poco significativas. (Lucio et al., 2001). Finalmente es importante enfatizar que el MMPI-2 aparece más apropiado que el MMPI original para evaluar población mexicana.

\section{Procedimiento}

Con los debidos permisos obtenidos de la Judicatura y de las autoridades regentes en cada centro penitenciario para realizar esta investigación, se consiguió entrevistar a los internos con un mínimo de garantías de comodidad y de colaboración. Algunos protocolos fueron administrados de forma personal o en pequeños grupos, según la disponibilidad de los recursos que disponía el Centro. Los participantes de ambos grupos aceptaron colaborar voluntariamente en este estudio, a quienes se les explicó la naturaleza de su participación, asegurando la confidencialidad de los datos recogidos y la exclusividad de su uso para fines de investigación (Consentimiento Informado). 
Se aplicó el Minnesota Multiphasic Personality Inventory-2 en su versión adaptada a la población mexicana (Lucio, Reyes-Laguna \& Scott, 1994). Uno de los problemas latentes en este tipo de personas es la credibilidad de la información que aportan, ya que el mismo contexto carcelario impide ofrecer una información sincera y veraz por las "consecuencias" que pueda tener. Al trabajar en este contexto se siguieron las recomendaciones seńaladas por Megargee (2006a) acerca de los puntos de corte de las escalas de Validez del MMPI-2 para detectar protocolos incompletos, respuestas aleatorias o incoherentes, fundamentalmente con VRIN y TRIN, antes que con las F (Infrecuencia), L (Mentira) y K (Defensividad). Se descartaron todos aquellos protocolos que el número de ítems No-sé/No-contesta (?) fuera $\geq 30, \mathrm{~L} \geq 80 \mathrm{~T}, \mathrm{~F} \geq 90 \mathrm{~T}, \mathrm{Fb}$ $\geq 80 \mathrm{~T}$ y $\mathrm{K} \geq 80 \mathrm{~T}$, y que los valores de VRIN y/o TRIN estuvieran entre 65T-79T (inclusive). Con ello se logró mayor fiabilidad y precisión en las respuestas al MMPI-2. Todas las puntuaciones obtenidas fueron baremadas y tratadas en sus análisis estadísticos en puntuaciones " $T$ " de acuerdo con la baremación mexicana del MMPI-2 (Lucio, ReyesLaguna \& Scott, 1994). Para este estudio, se homogeneizaron las muestras en número y género de un total de 934 No-delictivo (tomando como referencia los 287 Homicidas: 215 varones y 72 mujeres) que, de forma totalmente aleatoria, se extrajeron una vez filtrados del valor de las escalas de Validez.

\section{Resultados}

Se muestran todas aquellas escalas del MMPI-2 que, al contrastar sus diferencias estadísticamente significativas, han presentado un valor considerado como "grande" ( $\geq .90)$ en el tamaño del efecto $d$ de Cohen (1988). Posteriormente se comprobaron, a través de los índices de la curva ROC, la precisión diagnóstica de cada una de estas escalas.

En la Tabla 1 se exponen las diferencias habidas entre los dos grupos: los que no cometieron delito alguno y los condenados por homicidio. Se ha de observar que existen algunas escalas cuyos valores 
resultaron ser superiores en el grupo No-delictivo, como sucede con las escalas de Responsabilidad social (Re), Fuerza del Yo (Es), Dominancia (Do), Rol Masculino (GM) y Rol Femenino (GF).

\section{Tabla 1}

Diferencia de medias, significación y tamaño del efecto* entre grupos en las Escalas de Validez, Clínicas básicas, de Contenido y Suplementarias

\begin{tabular}{|c|c|c|c|c|c|}
\hline Escalas del MMPI-2 & Grupos & $M$ & $D E$ & $\mathrm{p}$ & $d$ \\
\hline \multirow{2}{*}{ Infrecuencia } & No-delictivo & 43.45 & 7.81 & \multirow{2}{*}{.00} & \multirow{2}{*}{1.52} \\
\hline & Homicida & 57.24 & 10.18 & & \\
\hline \multirow{2}{*}{ Infrecuencia posterior } & No-delictivo & 44.22 & 6.95 & \multirow{2}{*}{.00} & \multirow{2}{*}{1.20} \\
\hline & Homicida & 55.22 & 10.91 & & \\
\hline \multirow{2}{*}{ Desviación psicopática } & No-delictivo & 48.31 & 8.22 & \multirow{2}{*}{.00} & \multirow{2}{*}{1.00} \\
\hline & Homicida & 58.24 & 11.34 & & \\
\hline \multirow{2}{*}{ Esquizofrenia } & No-delictivo & 46.61 & 8.47 & \multirow{2}{*}{.00} & \multirow{2}{*}{.90} \\
\hline & Homicida & 55.01 & 10.33 & & \\
\hline \multirow{2}{*}{ Paranoia } & No-delictivo & 50.74 & 7.93 & \multirow{2}{*}{.00} & \multirow{2}{*}{.79} \\
\hline & Homicida & 58.39 & 11.08 & & \\
\hline \multirow{2}{*}{ Introversión social } & Homicida & 44.77 & 8.09 & \multirow{2}{*}{.00} & \multirow{2}{*}{.90} \\
\hline & Homicida & 52.25 & 8.60 & & \\
\hline \multirow{2}{*}{ Depresión } & No-delictivo & 44.03 & 8.26 & \multirow{2}{*}{.00} & \multirow{2}{*}{1.44} \\
\hline & Homicida & 56.79 & 9.41 & & \\
\hline \multirow{2}{*}{ Preocupaciones por la salud } & No-delictivo & 45.01 & 7.76 & \multirow{2}{*}{.00} & \multirow{2}{*}{1.09} \\
\hline & Homicida & 54.93 & 10.20 & & \\
\hline \multirow{2}{*}{ Cinismo } & No-delictivo & 42.75 & 8.42 & \multirow{2}{*}{.00} & \multirow{2}{*}{1.32} \\
\hline & Homicida & 55.02 & 10.09 & & \\
\hline \multirow{2}{*}{ Prácticas antisociales } & No-delictivo & 41.63 & 7.83 & \multirow{2}{*}{.00} & \multirow{2}{*}{1.35} \\
\hline & Homicida & 54.62 & 11.17 & & \\
\hline
\end{tabular}


La contribución del MMPI-2 a la predicción del riesgo de violencia / Ampudia, Sánchez, Jiménez

\begin{tabular}{|c|c|c|c|c|c|}
\hline Escalas del MMPI-2 & Grupos & $M$ & $D E$ & $\mathrm{p}$ & $d$ \\
\hline \multirow{2}{*}{ Problemas familiares } & No-delictivo & 43.57 & 8.03 & \multirow{2}{*}{.00} & \multirow{2}{*}{.90} \\
\hline & Homicida & 51.26 & 9.08 & & \\
\hline \multirow{2}{*}{ Dificultades en el trabajo } & No-delictivo & 44.67 & 8.66 & \multirow{2}{*}{.00} & \multirow{2}{*}{.91} \\
\hline & Homicida & 52.41 & 8.32 & & \\
\hline \multirow{2}{*}{ Negatividad al tratamiento } & No-delictivo & 43.65 & 8.64 & \multirow{2}{*}{.00} & \multirow{2}{*}{1.07} \\
\hline & Homicida & 53.25 & 9.31 & & \\
\hline \multirow{2}{*}{ Ansiedad (Welsh) } & No-delictivo & 44.76 & 8.19 & \multirow{2}{*}{.00} & \multirow{2}{*}{1.02} \\
\hline & Homicida & 53.49 & 8.97 & & \\
\hline \multirow{2}{*}{ Fuerza del yo } & No-delictivo & 52.84 & 7.60 & \multirow{2}{*}{.00} & \multirow{2}{*}{1.16} \\
\hline & Homicida & 42.75 & 9.58 & & \\
\hline \multirow{2}{*}{ Alcoholismo de MacAndrew } & No-delictivo & 45.30 & 8.14 & \multirow{2}{*}{.00} & \multirow{2}{*}{1.40} \\
\hline & Homicida & 59.23 & 11.52 & & \\
\hline \multirow{2}{*}{ Dominancia } & No-delictivo & 63.12 & 17.70 & \multirow{2}{*}{.00} & \multirow{2}{*}{1.39} \\
\hline & Homicida & 42.77 & 10.13 & & \\
\hline \multirow{2}{*}{ Responsabilidad social } & No-delictivo & 57.02 & 8.86 & \multirow{2}{*}{.00} & \multirow{2}{*}{1.30} \\
\hline & Homicida & 44.09 & 10.89 & & \\
\hline \multirow{2}{*}{ Desajuste profesional } & No-delictivo & 43.69 & 8.34 & \multirow{2}{*}{.00} & \multirow{2}{*}{.95} \\
\hline & Homicida & 51.89 & 8.86 & & \\
\hline \multirow{2}{*}{ Rol masculino } & No-delictivo & 55.14 & 10.16 & \multirow{2}{*}{.00} & \multirow{2}{*}{1.07} \\
\hline & Homicida & 44.15 & 10.36 & & \\
\hline \multirow{2}{*}{ Rol femenino } & No-delictivo & 53.56 & 8.72 & \multirow{2}{*}{.00} & \multirow{2}{*}{.92} \\
\hline & Homicida & 44.32 & 11.25 & & \\
\hline \multirow{2}{*}{$\begin{array}{l}\text { Trastorno de estrés } \\
\text { Postraumático (Keane) }\end{array}$} & No-delictivo & 44.69 & 8.34 & OQ & 115 \\
\hline & Homicida & 55.28 & 10.07 & .00 & 1.15 \\
\hline
\end{tabular}

* Con diferencias del tamaño del efecto $d \geq 0.90$ (excepto con Pa). 
La Tabla 2 muestra, a través de la curva ROC, los principales índices de precisión diagnóstica solo de las escalas que resultaron obtener una diferencia "grande" en el tamaño del efecto de Cohen ( $\geq .90)$ al comparar los dos grupos de este estudio (Tabla 1). Los resultados muestran la existencia de varias escalas de Contenido y Suplementarias que obtienen mayor precisión diagnóstica global (AUC $\geq .80)$ que las escalas Clínicas básicas (DEP, HEA, CYN, ASP, PK, Mac-R; Es, Do, Re).

\section{Tabla 2}

Indices de precisión diagnóstica aportados por la curva ROC con las escalas* del MMPI-2

\begin{tabular}{|c|c|c|c|c|c|c|}
\hline $\begin{array}{l}\text { Escalas del } \\
\text { MMPI-2 }\end{array}$ & AUC & SE & $95 \% \mathrm{CI}$ & Sens. $\%$ & Esp.\% & $\begin{array}{c}\text { Punto de } \\
\text { corte }\end{array}$ \\
\hline F & .863 & .0152 & $.832-.890$ & 82.58 & 77.35 & $>46$ \\
\hline $\mathrm{Fb}$ & .813 & .0179 & $.778-.844$ & 78.40 & 71.78 & $>44$ \\
\hline $\mathrm{Pd}$ & .760 & .0200 & $.723-.794$ & 62.37 & 78.75 & $>53$ \\
\hline Sc & .741 & .0207 & $.703-.776$ & 59.93 & 79.79 & $>51$ \\
\hline $\mathrm{Pa}$ & .702 & .0218 & $.663-.740$ & 54.01 & 82.58 & $>56$ \\
\hline $\mathrm{Si}$ & .744 & .0203 & $.707-.780$ & 78.75 & 57.14 & $>44$ \\
\hline DEP & .848 & .0165 & $.816-.876$ & 80.49 & 77.00 & $>48$ \\
\hline HEA & .790 & .0189 & $.754-.822$ & 79.09 & 67.94 & $>47$ \\
\hline CYN & .832 & .0168 & $.799-.862$ & 87.11 & 63.76 & $>44$ \\
\hline ASP & .833 & .0164 & $.800-.863$ & 74.91 & 75.61 & $>46$ \\
\hline FAM & .744 & .0204 & $.706-.779$ & 69.69 & 70.03 & $>45$ \\
\hline WRK & .759 & .0203 & $.722-.793$ & 69.34 & 74.22 & $>48$ \\
\hline TRT & .790 & .0192 & $.755-.823$ & 70.73 & 79.44 & $>47$ \\
\hline A & .783 & .0194 & $.747-.816$ & 78.40 & 68.64 & $>46$ \\
\hline Es & .800 & .0183 & $.764-.832$ & 70.73 & 76.66 & $\leq 48$ \\
\hline Mac-R & .839 & .0164 & $.807-.868$ & 67.60 & 86.41 & $>53$ \\
\hline
\end{tabular}




\begin{tabular}{ccccccc}
\hline $\begin{array}{c}\text { Escalas del } \\
\text { MMPI-2 }\end{array}$ & AUC & SE & $95 \%$ CI & Sens.\% & Esp.\% & $\begin{array}{c}\text { Punto de } \\
\text { corte }\end{array}$ \\
\hline Do & .848 & .0162 & $.816-.876$ & 84.67 & 75.26 & $\leq 52$ \\
Re & .823 & .0171 & $.789-.853$ & 78.40 & 73.87 & $\leq 50$ \\
Mt & .752 & .0199 & $.725-.796$ & 71.08 & 69.69 & $>45$ \\
GM. & .784 & .0189 & $.748-.817$ & 77.35 & 67.94 & $\leq 51$ \\
GF & .736 & .0205 & $.698-.772$ & 52.96 & 83.97 & $\leq 45$ \\
PK & .806 & .0181 & $.771-.838$ & 78.75 & 70.03 & $>46$ \\
\hline
\end{tabular}

* Con diferencias del Tamaño del efecto $\mathrm{d} \geq 0.90$ (excepto Pa).

Notas. AUC: Área bajo la curva; SE: Error Estándar; CI: Intervalo de Confianza; Sens: Sensibilidad; Esp.: Especificidad. F = Infrecuencia; Fb: Infrecuencia posterior; Pd: Desviación psicopática; Sc: Esquizofrenia; Pa: Paranoia; Si: Introversión social; DEP: Depresión; HEA: Preocupaciones por la salud; CYN: Cinismo; ASP: Prácticas antisociales; Mac-R: Alcoholismo de MacAndrew; Re: Responsabilidad social; FAM: Problemas familiares; WRK: Dificultades en el trabajo; TRT: Negatividad al tratamiento; A: Ansiedad (Welsh); Mt: Desajuste profesional; PK: Trastorno por estrés postraumático (Keane); Es: Fuerza del yo; Do: Dominancia; GM: Rol masculino; GF: Rol femenino.

La Figura 1 presenta seis escalas del MMPI-2 implicadas en la predicción del riesgo de violencia, en las que se pueden apreciar las diferentes curvas que varían según el punto de corte elegido: dos escalas detectoras de conducta antisocial (Pd y ASP), otras dos escalas detectoras de un realidad errática (Sc y Pa) y la escala de alcoholismo de MacAndrew (Mac-R) y la de Cinismo (CYN) como posible catalizadora de la violencia. 


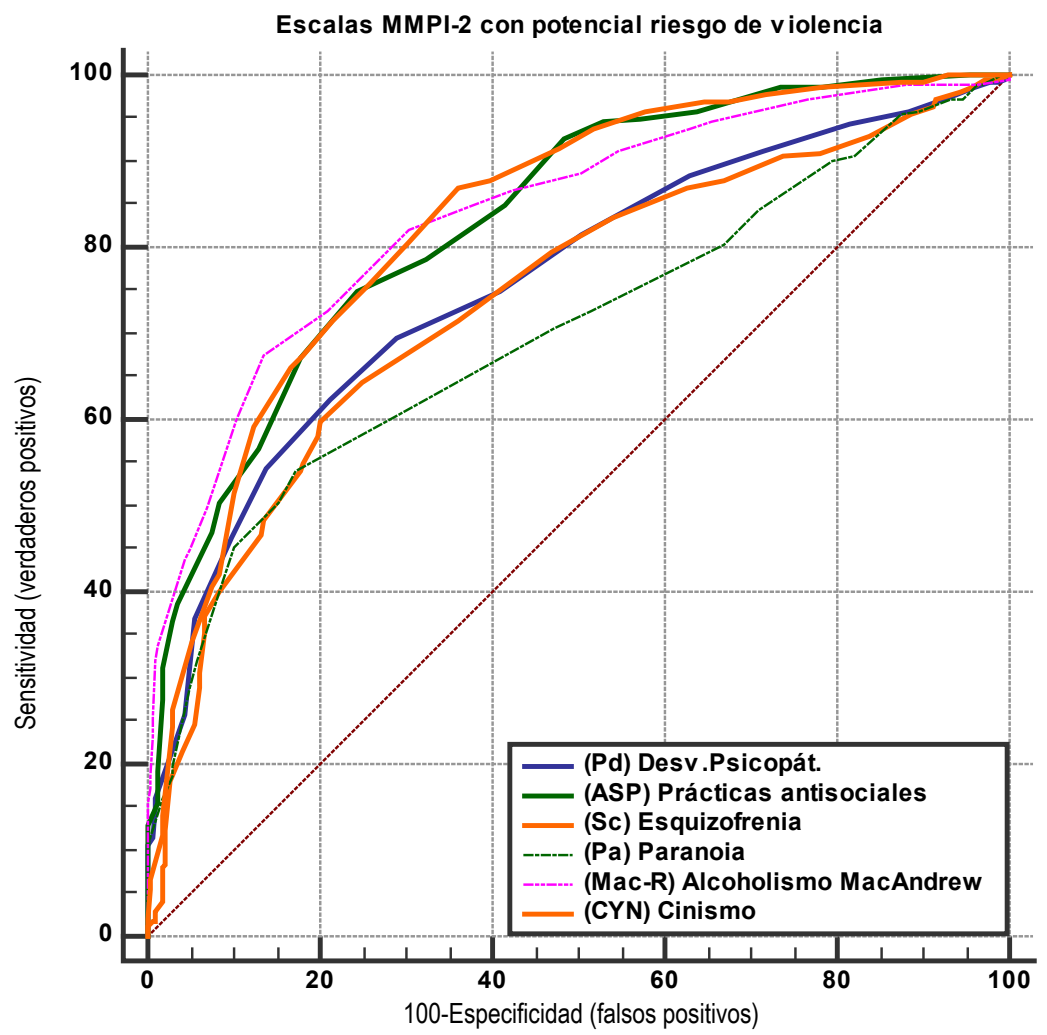

Figura 1. Seis escalas del MMPI-2 implicadas en la predicción del riesgo de violencia.

\section{Discusión}

Somos plenamente conscientes de que el MMPI/MMPI-2 no es la técnica más adecuada para predecir por sí misma el riesgo de violencia, ni siquiera en contextos como el carcelario (Heilbrum \& Heilbrum, 
2009), a pesar de las numerosas investigaciones existentes, porque la violencia interpersonal está muy condicionada por factores situacionales que el MMPI no puede evaluar y debe apoyarse de otras informaciones. Existen otras técnicas más específicas que podrían aportar mayor precisión y fiabilidad, tales como la Historical/Clinical/Risk Management-20 (HCR-20, Webster, Douglas, Eaves \& Hart, 1997), la Escala de Valoración de la Conducta Violenta y Riesgo de Reincidencia (EVCV-RR, Jiménez, Sánchez, Merino y Ampudia, 2014), la Violent Risk Appraisal Guide (VRAG, Quinsey, Harris, Rice \& Cormier, 2006) o la Classification of Violence Risk (COVR, Monahan et al., 2005), entre otros. Sin embargo, también somos conscientes de que el MMPI-2 podría aportar otra información diferente ofreciendo una visión global de factores predisponentes y/o relacionados con la personalidad violenta: psicopatología, trastornos del pensamiento, desajustes sociofamiliares o desajustes emocionales.

Los resultados de este estudio, en general, son consistentes con los referidos por la mayor parte de los autores que han utilizado el MMPI-2 para evaluar el riesgo de violencia (Butcher, 2013; Butcher, Hess, Greene \& Nelson, 2015; Grover, 2011; Jones, Beidleman, y Fowler, 1981; Megargee, 1977, 2006a, 2006b; Nieberding, Moore, y Dematatis, 2002; Pennuto, 2010; Lanyon, 1993; Rader, 1977) con las principales escalas del MMPI-2: Pd, Sc, Pa, Mac-R. Observamos que este conjunto de variables no se ajusta totalmente a la tétrada psicótica (Pa, Sc, Ma, Pt), pero sí tiene variables psicóticas (Sc y Pa) que, efectivamente, pueden hacer más peligrosa su conducta ya antisocial $(\mathrm{Pd})$. Y también se ha encontrado, en este estudio, aportaciones interesantes que pueden ańadir una importante contribución a la predicción del riesgo de violencia. A las variables que denotan problemas de conducta, tal como la Pd (Desviación Psicopática), se ha sumado la escala de Prácticas antisociales (ASP) con un gran tamaño del efecto $(d=1.35)$ y una precisión diagnóstica global aceptable (AUC $=.833)$; la responsabilidad social (Re) también ha mostrado una gran diferencia, al ser su valor muy inferior en comparación con el grupo No-delictivo, tanto en el tamańo del efecto $(d=1.30)$ como en la precisión diagnóstica 
$(\mathrm{AUC}=.823)$; es decir, se está constatando la incapacidad para aceptar responsabilidades sociales por parte del grupo de Homicidas.

El Cinismo (CYN), propio de personas que recurren al engaño, a la hipocresía y a la manipulación de los demás para beneficio propio y justificar sus comportamientos de explotación con la racionalización de que los demás son igualmente egoístas, deshonestos, y amorales (Butcher et al., 2015), es otra de las escalas que han caracterizado a la personalidad del Homicida, con una gran diferencia en el tamańo del efecto $(d=1.32)$ y una buena precisión diagnóstica global (AUC $=$ .832). Sin embargo, nos han llamado la atención los resultados obtenidos por la escala de Introversión Social (Si), ya que no es una escala que aparezca normalmente en los estudios con delincuentes en contextos carcelarios (Butcher et al., 2015; Megargee, 2006b). Con una diferencia del tamaño del efecto $(d=0.90)$ y una precisión diagnóstica global $(A U C=.744)$, la Introversión social $(\mathrm{Si})$, refiere una grave ausencia de contacto con los demás, refugiándose en sí mismo como una forma de protección de las posibles agresiones de los demás.

En un contexto carcelario, especificado por el crimen organizado, las mafias y las bandas carcelarias, reduciendo y determinando el contacto con los demás, pueden estar condicionando determinadas conductas y características psicológicas personales, tales como el silencio, el miedo, relacionarse solo con los de su banda, el mutismo, el sentimiento de sospecha y desconfianza mezclados con la percepción y creencia de tipo paranoica que se han encontrado entre los reclusos de este estudio.

Con las elevadas puntuaciones mostradas por la escala WRK, también parece que los Homicidas están experimentando una amplia variedad de problemas que pueden interferir con su capacidad para llevar a cabo sus responsabilidades de trabajo (Butcher et al., 2015). Una diferencia importante con el grupo No-delictivo les caracteriza con un tamaño del efecto $(d=.91)$ y una precisión diagnóstica (AUC $=.759)$. La posibilidad de tratamiento, mediante la escala TRT, no parece posible $(d=1.07 ; \mathrm{AUC}=.790)$, haciéndoles caracterizarse como parásitos de 
la sociedad para vivir a costa de los demás y con una gran ausencia de metas realistas para formarse en el futuro e integrase en la sociedad.

La adicción al alcohol durante mucho tiempo ha sido reconocida como uno de los factores precipitantes de la violencia criminal (Holcomb \& Adams, 1985). La investigación ha encontrado consistentemente una fuerte relación entre la adicción al alcohol y homicidio, con más del $50 \%$ de los asesinos intoxicados cuando matan a sus víctimas (Holcomb y Anderson, 1983). En este estudio se ha constatado igualmente el consenso de los investigadores cuando el alcohol se puede convertir en un catalizador de la expresión de violencia y, en la escala de consumo del alcohol de MacAndrew (Mac-R), una gran diferencia en el tamaño del efecto $(d=1.40)$ y en su precisión diagnóstica (AUC = 0.839), al comparar a los Homicidas con las personas que no han cometido delito alguno. Al ser la violencia situacional (Heilbrum \& Heilbrum, 2009), se hace necesario tenerla en cuenta y, en determinados contextos, incluso carcelarios, esta conducta se puede manifestar de diversas formas y el MMPI-2 puede ser reflejo de esta situación.

Los resultados aportados en este estudio, referentes a las escalas de Problemas de salud (HEA), de Depresión (DEP), de Ansiedad (A), de Trastorno por Estrés Postraumático (PK), y de Introversión social $(\mathrm{Si})$, podrían estar relacionados con las mismas condiciones carcelarias mexicanas y no tanto ser características de la personalidad del Homicida. Una serie de encuestas realizadas a los reclusos mexicanos durante varios años (Azaola \& Bergman, 2003, 2009; Bergman, Azaola \& Magaloni, 2005, 2006; Pérez \& Azaola, 2012) nos hacen ver la situación en la que se encuentran los reclusos mexicanos. El contexto carcelario en México tiene sus propias peculiaridades. Según las estadísticas de la Secretaría de Gobernación (2013), existen 420 centros penitenciarios y un total de 242.754 internos en dichas instalaciones que ocupan un espacio diseńado para 195.278, lo cual indica que la ocupación alcanza $124.3 \%$ con sobrepoblación en 220 de un total de 420 centros penitenciarios. Esta sobrepoblación (Villanueva, 2015) está generando, a su vez, otros muchos problemas: bandas organizadas, falta de control de la misma cárcel, ingobernabilidad, insuficiencia/carencia de los 
servicios básicos, inadecuada clasificación de los internos, menor integración de sus responsabilidades de los empleados y, en definitiva, falta deoportunidades reales de acceso a los medios para lograr la reinserción social efectiva.

Llamas (2013) nos aporta datos con mayor precisión: el 56,3\% del total de los internos se encuentran sentenciados, mientras que el $43,7 \%$ son preventivos; es decir, más de 100.000 personas se encuentran en espera de ser juzgadas, con la peculiaridad de que algunos podrían resultar absueltos. Igualmente es preocupante la elevada población de reincidentes que, entre 2005 y 2009, creció un 17\% (Azaola \& Bergman, 2009). Según las estadísticas de la Subsecretaría del Sistema Penitenciario (SSP): "En la actualidad hay 36.501 internos en los 13 penales de la Ciudad de México, de los cuales 14.158 son reincidentes, lo que representa 38.78\%" (Diario Excelsior; 4/02/2016).

Solís, Buen y Ley (2013) critican "las condiciones de vida en el interior de los penales que, en vez de permitir la reinserción de los sentenciados a la sociedad, favorecen la contaminación criminógena" (p. 5). Todo esto puede estar explicando, en este estudio, la aparición de algunas escalas del MMPI-2 peculiares de los reclusos mexicanos y que, generalmente, no suelen aparecer en otros estudios. Megargee (2006a) aconseja que los delincuentes que son encarcelados deberían ser examinados sobre tres aspectos: su salud mental, valorar los riesgos y sus necesidades.

En este estudio se han tomado los principales índices de precisión diagnóstica aportados la curva ROC para evaluar las escalas del MMPI-2. A través de sus índices, fundamentalmente, el Área Bajo la Curva (AUC; Area Under Curve), la Sensibilidad y la Especificidad, se ofrece una visión global de la exactitud diagnóstica proporcionando datos significativos acerca de la probabilidad de clasificar correctamente a un individuo mediante una variable determinada. La curva ROC (Figura 1) es un gráfico en el que se observan todos los pares Sensibilidad/Especificidad resultantes de la variación continua de los puntos de corte en todo el rango de resultados observados. En el eje de ordenadas se sitúa la Sensibilidad, o fracción de Verdaderos Positivos, y 
en el eje X se sitúa la fracción de los Falsos Positivos (1-Especificidad) (Burgueño et al., 1995).

El índice obtenido por el Área Bajo la Curva (AUC) nos muestra la probabilidad de clasificar correctamente un par de individuos, Homicida o No-delictivo, seleccionados al azar de la población, con los resultados obtenidos al aplicarles la prueba diagnóstica. Es, en definitiva, una medida global de la exactitud de la prueba para clasificar a los individuos. Cuanto más elevada, mayor exactitud. Así, el valor de AUC en $M a c-R=.839$, en el conjunto de Homicidas/No-delictivo (Tabla 2), significa que un individuo, seleccionado aleatoriamente del grupo de Homicidas, tendrá el $83.9 \%$ de las veces un valor mayor que un individuo elegido al azar del grupo No-delictivo.

Swets (1979) interpreta los valores de la AUC de la siguiente manera: entre $0.5-0.7$ indican baja exactitud, entre $.7-.9$ pueden ser útiles para algunos propósitos y un valor mayor de .9 indica exactitud alta. De todas las escalas del MMPI-2, en sus diversos conjuntos de variables, se eligieron aquellas escalas que la $\mathrm{AUC} \geq 0.700$ considerando que, a partir de este valor, la precisión diagnóstica es aceptable (Tabla 2). La capacidad de discriminación de la prueba diagnóstica se especifica estimando el Intervalo de confianza (CI) de la AUC: si el intervalo no incluye el valor de 0.5 , la prueba es capaz de discernir entre Homicidas y No-delictivo. Por convenio, la AUC es siempre $\geq .5$ porque sus valores están comprendidos entre .5 (si no existen diferencias en la distribución de resultados de la prueba entre los subgrupos delincuente y no-delincuentes) y 1.0 cuando existe separación perfecta entre las dos distribuciones.

Con la Sensibilidad de una prueba se obtiene la probabilidad (\%) de clasificar correctamente a un individuo cuya situación real sea definido como "positivo" (Homicida); detecta a los "verdaderos Homicidas". La Especificidad detecta la probabilidad (\%) de clasificar correctamente a un individuo cuya situación sea definida como "negativo" (No-delictivo); detecta a los "verdaderos No-delictivo". Por ejemplo, siguiendo con la referencia de la escala $M a c-R$ del conjunto de Homicidas/No-delictivo (Tabla 2), se puede ver que, con el punto de 
corte $>53$ (en puntuaciones T de la adaptación mexicana del MMPI-2), la Sensibilidad nos aporta la información de que clasificaría correctamente al $67.6 \%$ como verdaderos Homicidas y el resto (100-67.6 = $32.4 \%)$ serían considerados como falsos positivos. La Especificidad de esta misma escala $M a c-R$ diagnosticaría correctamente a un $86.4 \%$ de sujetos No-delictivo y al 13,6\% (100-86.4) resultaría ser falsos negativos. Con los valores mostrados por estos tres índices (AUC, Sensibilidad y Especificidad) se puede concluir que la precisión diagnóstica de la variable $M a c-R$ del MMPI-2, puede ser considerada como "moderada" (Swets, 1979). Se ha de advertir que si se toma otro punto de corte diferente, el binomio Sensibilidad/Especificidad variará. Hemos tomado como referencia la escala $M a c-R$, pero es un ejemplo de cómo interpretar cualquier escala de la Tabla 2.

¿Esto es mucho? Pues depende de cómo se interprete, en qué contexto y cuál es el riesgo que se está dispuesto a aceptar. No es lo mismo un contexto judicial o médico donde un porcentaje de falsos positivos o negativos pueda ser considerado como injustificable, que en otro contexto que se cuenta con información contrastada, objetiva o de experiencia profesional. Ya hemos comentado que el objetivo de este trabajo era el de poder apreciar la contribución que hace la técnica del MMPI-2 a la predicción del riesgo de violencia y nos hemos encontrado con unas diferencias importantes y unos valores de precisión diagnóstica muy interesantes.

Sin embargo, sería un grave error evaluar con una sola prueba (por mucha precisión diagnóstica que tenga) y arriesgarse a emitir un diagnóstico. Algunos investigadores (Kozol, Boucher \& Garofalo, 1972; Megargee, 1976) han señalado que es casi imposible predecir con precisión comportamientos violentos únicamente sobre la base de los resultados de las pruebas de personalidad debido a que tales comportamientos se encuentran influenciados, en gran medida, por la gran variedad de factores que intervienen en la manifestación de la violencia, siendo un fenómeno muy complejo y multicausal.

Somos conscientes de las limitaciones que hemos encontrado en la elaboración de este estudio. Por una parte, el elevado porcentaje (43.7\%; 
Llamas, 2013) de reclusos preventivos (en espera de juicio) pero no sentenciados por homicidio en las cárceles mexicanas; por otra, la elevada masificación (124.3\% con sobrepoblación en 220 de un total de 420 centros penitenciarios; Secretaría de Gobernación, 2013) que genera un comportamiento de supervivencia y que puede estar afectando a los datos obtenidos. Con todo ello, hemos llegado a la conclusión de que el Minnesota Multiphasic Personality Inventory-2 (MMPI-2) es una técnica psicológica que puede contribuir positivamente a la predicción del riesgo de violencia.

\section{Referencias}

Ampudia, A., Sánchez, G. \& Jiménez, F. (2017). Precisión diagnóstica del MMPI-2 con la personalidad delictiva: un análisis con la curva ROC. Revista de Psicología, 35, 167-192. https://doi. org/10.18800/psico.201701.006

Andrés Pueyo, A. \& Redondo, S. (2007). Predicción de la violencia: entre la peligrosidad y la valoración del riesgo de violencia. Papeles del Psicólogo, 28, 157-173.

Azaola, E. \& Bergman, M. (2003). El sistema penitenciario mexicano. Project on Reforming the Administration of Justice in Mexico, Center for U.S.-Mexican Studies, UC San Diego.

Azaola, E. \& Bergman, M. (2009). Delincuencia, marginalidady desempeño institucional. Resultados de la tercera encuesta a población en reclusión en el Distrito Federal y Estado de México. México, DF: Centro de investigación y Docencia Económicas (CIDE).

Bergman, M., Azaola, E. \& Magaloni, A.-L. (2005). Encuesta a la población en reclusión en el Distrito Federal y Estado de México. México, DF: Centro de investigación y Docencia Económicas (CIDE).

Bergman, M., Azaola, E. \& Magaloni, A.-L. (2006). Delincuencia, marginalidad y desempeño institucional. Resultados de la segunda encuesta a población en reclusión en el Distrito Federal y el Estado 
de México. México, DF: Centro de Investigación y Docencia Económicas (CIDE).

Boscan, D. C., Penn, N. E., Velasquez, R. J., Savino, A. V., Maness, P., Guzman, M. \& Reimann, J. (2002). MMPI-2 Performance of Mexican Male University Students and Prison Inmates. Journal of Clinical Psychology, 58, 465-470. https://doi.org/10.1002/ jclp. 1156

Burgueño, M. J., García Bastos, J. L. \& González Buitrago, J. M. (1995). Las curvas ROC en la evaluación de las pruebas diagnósticas. Medicina clínica, 104, 661-670.

Butcher, J. N. (2013). 25 Highlights of Using the MMPI/MMPI-2 with Criminal Offenders. https://doi.org/10.1037/14571-000

Butcher, J. N. Hess, G. A., Greene, R. L. \& Nelson, L. D. (2015). Using the MMPI-2 in Forensic Assessment. American Psychological Association (APA).

Cohen, J. (1988). Statistical Power Analysis for the Behavioral Sciences (2a.Ed). LEA. New Jersey.

Cunningham, M. D., Sorensen J. R. \& Reidy, T. J. (2005). An actuarial model for Assessment of prison violence risk among maximum security inmates. Assessment, 12, 40-49. http://dx.doi. org/10.1177/1073191104272815

Derzon, J. H. (2001). Antisocial behaviour and the prediction of violence: a meta-analysis. Psychology in the Schools, 3, 93-106. http://dx.doi.org/10.1002/pits.1002

Diario Excelsior (2016). "Más de 14.000 presos están por reingreso en reclusorios de la Ciudad de México. Obtenido en fecha de 29/02/2016. http://www.excelsior.com.mx/ comunidad/2016/02/02/1072503.

Eronen, M. Hakolaq, P. \& Tiihonen (1996). Mental disorders and homicidal behavior in Filand. Archives of General Psychiatry, 53, 497-501. http://dx.doi.org/10.1001/ archpsyc. 1996.01830060039005

Fry, F. D. (1949). A study of the personality traits of college students, and of state prison inmates as measured by the Minnesota 
Multiphasic Personality Inventory. Journal of Psychology: Interdisciplinary and Applied, 28, 439-449. doi.org/10.1080/00223 980.1949.9916021

Gardner, W., Lidz, C. W., Mulvey E. P. \& Shaw, E. C. (1996). Clinical versus predictions of violence inpatients with mental Illness. Journal of Consulting and Clinical Psychology, 64, 602-609. http://dx.doi.org/10.1037/0022-006X.64.3.602

Grisso, T. \& Appelbaum, P. (1992). Is it unethical to offer predictions of future violent behavior? Law and Human Behavior, 16, 621633. http://dx.doi.org/10.1007/BF01884019

Grover, B. L. (2011). The Utility of MMPI-2 Scores with a Correctional Population \& Convicted Sex Offenders. Psychology, 2, 638-642. doi.org/10.4236/psych.2011.26098

Gutiérrez, J.-R. \& Portillo, C.-B. (2014). La violencia delincuencial asociada a la salud mental en la población salvadoreńa. Revista de Psicología, 32, 3-37.

Guy, E., Platt, J. J., Zwerling, I. \& Bullock, S. (1985). Mental health status of prisoners in an urban jail. Criminal Justice \& Behavior, 12, 29-53. doi.org/10.1177/0093854885012001004

Hathaway, S. R. \& McKinley, J. C. (1967). Minnesota Multiphasic Personality Inventory (rev.ed.). New York: Psychological Corporation.

Heilbrum, K. (2009). Evaluation for risk of violence in adults. New York: Oxford University Press.

Holcomb, W. R. \& Adams, N. A. (1985). Personality mechanisms of alcohol-related violence. Journal of Clinical Psychology, 41, 714-722. http://dx.doi. org/10.1002/1097-4679(198509)41:5\%3C714::AIDJCLP2270410522\%3E3.0.CO;2-0

Holcomb, W. R. \& Anderson, W. P. (1983). Alcohol and multiple drug abuse in accused murderers. Psychological Reports, 52, 159-164. http://dx.doi.org/10.2466/pr0.1983.52.1.159

Jiménez, F., Sánchez, G., Merino, V. \& Ampudia, A. (2014). Evaluación de la peligrosidad en la población penitenciaria. Valoración 
del riesgo de violencia y de su incidencia (EVCV-RR). Salamanca: Ratio Legis.

Jones, T., Beidleman, W. B. \& Fowler, R. D. (1981). Differentiating violent and nonviolent prison inmates by use of selected MMPI scales. Journal of Clinical Psychology, 37, 673-678. doi.org/10.1002/1097-4679(198107)37:3\%3C673::AIDJCLP2270370340\%3E3.0.CO;2-P

Kozol, H. L., Boucher, R. J. \& Garofalo, R. F. (1972). The Diagnosis and Treatment of Dangerousness. Crime \& Delinquency, 18, 371-392. http://dx.doi.org/10.1177/001112877201800407

Krug, E. G., Dahlberg, L. L., Mercy, J. A., Zwi, A. B. \& Lozano, R. (2002). World report on violence and Health. Geneve. WHO.

Lanyon, R. I. (1993). Validity of MMPI sex offender scales with admitters and no admitters Psychological Assessment, 5, 302-306. doi. org/10.1037/1040-3590.5.3.302

Llamas, M. (2013). El sistema penitenciario en cifras. SinEmbargo. Opinión. Recuperado el 25/10/2015 de http//www.sinembargo. mx./opinion/28-07-2013/16235

Loza, W. (2003). Predicting violent and non-violent recidivism of incarcerated male offenders. Aggression and violent behaviour, 8, 175-203. http://dx.doi.org/10.1016/S1359-1789(01)00055-6

Lucio, E., Reyes-Lagunes, I. \& Scott, R. L. (1994). MMPI-2 for Mexico: Translation and adaptation. Journal of Personality Assessment, 63, 105-116. doi.org/10.1207/s15327752jpa6301_9

Lucio, E.M., Ampudia, A., Durán, C., Leon, I. \& Butcher, J.N, (2001). Comparisons of Mexican and American norms of the MMPI-2. Journal of Clinican Psychology, 57, 1459-1468.

Lucio G. M., E., Pérez, J. M. \& Ampudia, A. (1997). Un estudio de confiabilidad test-retest del MMPI-2 en un grupo de estudiantes mexicanos. Revista Mexicana de Psicología, 14(1), 55-62.

Meehl, P. (1954). Clinical versus statistical prediction: A theoretical analysis and review of the evidence. Minneapolis, MN: University of Minnesota Press. https://doi.org/10.1037/11281-000 
Megargee, E. I. (1976). The prediction of dangerous behaviour. Criminal Justice and Behavior, 3, 3-22. http://dx.doi. org/10.1177/009385487600300101

Megargee, E. I. (1997). Using the Megargee MMPI-based classification system with the MMPI-2s of female prison inmates. Psychological Assessment, 9, 75-82. doi.org/10.1037/1040-3590.9.2.75

Megargee, E. I. (2006a). Use of the MMPI-2 in correctional settings. En J. N. Bucther (Ed.), MMPI-2: A practitioner's guide (pp. 327360). Washington, DC: American Psychological Association (APA).

Megargee, E. I. (2006b). Using the MMPI-2 in criminal justice and correctional settings. Minneapolis, MN: University of Minnesota Press. doi.org/10.1037/0022-006X.53.6.874

Megargee, E. I., Mercer, S. J. \& Carbonell, J. L. (1999). MMPI-2 with male and female state and federal prison inmates. Psychological Assessment, 11, 177-185. http://dx.doi. org/10.1037/1040-3590.11.2.177

Monahan, J. (1981). Predicting violent behavior: An assessment of clinical techniques. Beverly Hill. CA. Sage.

Nieberding, R. J., Moore, J. T. \& Dematatis, A. P. (2008). Psychological Assessment of Forensic Psychiatric Outpatients. Assessment, $15,242-255$.

Oliver, W. \& Mosher, D. (1968). Psychopathology and guilt in heterosexual and subgroups of homosexual reformatory inmates. Journal of Abnormal Psychology, 73, 323-329. http://dx.doi. org/10.1037/h0026115

Otto, R. K. (2000). Assessing and managing violence risk in outpatient setting. JournalofClinicalPsychology, 56,1239-1262.http://dx.doi. org/10.1002/1097-4679(200010)56:10\%3C1239::AIDJCLP2\%3E3.0.CO;2-J

Panton, J. H. (1959). The response of prison inmates to seven new MMPI scales. Journal of Clinical Psychology, 15, 196-197. http:// dx.doi.org/10.1002/1097-4679(195904)15:2\%3C196::AIDJCLP2270150222\%3E3.0.CO;2-E 
Panton, J. H. (1962b). The identification of habitual criminalist with the MMPI. Journal of Clinical Psychology, 2, 133-136. http:// dx.doi.org/10.1002/1097-4679(196204)18:2\%3C133::AIDJCLP2270180207\%3E3.0.CO;2-2

Pavelka, F. L. (1986). Psychosocial characteristics of parolees in forensic social work. Journal of Psychiatry \& Law, 14, 217-223. https:// doi.org/10.1177/0093185386014001-210

Pennuto, T. O. (2010). Murder and the MMPI-2: The Necessity of Knowledgeable Legal Professionals. Golden Gate University Law Review, 34, 340-391.

Pérez, C. C. \& Azaola, E. (2012). Resultados de la primera encuesta realizada a población interna en Centros Federales de Readaptación Social. México: (CIDE).

Rader, C. M. (1977). MMPI profile types of exposers, rapists, and assaulters in a court services population. Journal of Consulting and Clinical Psychology, 45, 61-69. https://doi. org/10.1037/0022-006X.45.1.61

Secretaría de Gobernación (2013). "Estadísticas del Sistema Penitenciario Nacional', fechade acceso: 2 de julio de 2013, http://www.ssp.gob. $\mathrm{mx} /$ portalWebApp/ShowBinary?nodeId=/BEA\%20 Repository/ 365162//archivo.

Solís, L. Buen, N. \& Ley, S. (2013). La cárcel en México: ¿para qué?. México evalúa, Centro de Análisis de Políticas Públicas.

Soria, M. A., Armadans, I., Viñas, M. R. y Yepes, M. (2009). Homicide and domestic violence. Are there different Psychological profiles mediated by previous violence exerted on the victim? The European Journal of Psychology Applied to Legal Context, 1, 205-220.

Swets, J. A. \& Pickett, R. M. (1982). Evaluation of diagnostic systems: methods from signal detection theory. New York: Academic Press.

Torrubia, R. (2004). Predicción de la violencia. Trabajo presentado al I Congreso de Psicología Jurídica en Red. Colegio Oficial de Psicólogos: Madrid.

Villanueva, C. R. (2015). "La Sobrepoblación en los Centros Penitenciarios de la República Mexicana. Análisis y Pronunciamiento". 
Comisión Nacional de los Derechos Humanos. Comunicado de prensa CGCP/310/15. www.cndh.mx.

Webster, C. D., Douglas, K. S., Eaves, D. \& Hart, S. (1997b). HCR-20. Assessing risk for violence (version 2). Burnaby, British Columbia, Canadá: Simon Fraser University, Mental Health, Law and Policy Institute.

Webster, C. D., Hucker S. J. \& Bloom, H. (2002). Transcending the actuarial versus clinical polemic in assessing risk for violence. Criminal Justice and Behavior, 29, 659-665. http://dx.doi. org/10.1177/009385402236736

Zweig, M. H. \& Campbell, G. (1993). Receiver Operating Characteristic (ROC) plots: a fundamental evaluation tool in clinical medicine. Clinical Chemistry, 39, 561-577.

Recibido: 27 de enero, 2017 Revisado: 28 de setiembre, 2017 Aceptado: 24 de abril, 2018 